\title{
Skeletons of Glass Sponges as a Substrate for Creating Functional Composite Organosilicon Materials
}

\author{
Alexandr A. Karpenko ${ }^{1}$, Anatoliy L. Drozdov ${ }^{1,2}$ \\ 1. National Scientific Center of Marine Biology, Far Eastern Branch, Russian Academy of Sciences, 17 \\ Palchevsky str., Vladivostok 690041, Russia \\ E-mail: alex-karp@list.ru \\ 2. Far Eastern Federal University, 8 Sukhanov str., Vladivostok 690091, Russia \\ E-mail: anatoliyld@mail.ru
}

Received: 6 February 2019; Accepted: 2 March 2019; Available online: 20 April 2019

\begin{abstract}
The article describes a method of creating a three-dimensional organosilicon composite material based on the glass sponge spicules through a combination of soft etching and controlled mechanical action, nondestroying spicules. When in an alkaline medium, the material of the spicules' outer concentric layers is dissolved and silica passes into solution. After 20 to 30 days in alkaline medium, the silica is in excess and precipitates on organic components, forming a network of 300-500-nm thick organosilicon trabeculae, by which the spicules are fused into a single structure. The resulting composite material contains a mineral component of silica and an organic component. The results indicate that the natural organosilicon material can be reformed as a result of self-assembly into three-dimensional or flat structures.
\end{abstract}

Keywords: Hexactinellida; Glass sponges; Organosilicon spicules; Three-dimensional organosilicon composite material; Alkaline medium.

\section{Introduction}

The creation of new composite organosilicon materials with special physicochemical properties based on biomimetic approaches is actively discussed in scientific literature on materials science [1-6]. The unique physical properties of the spicules of glass sponges attract researchers from different fields [7]. These sponges are capable of organizing nanoscale building blocks into large-scale hierarchical structures - solid spicules. Particular attention has been given to the optical properties of these structures after a publication by Italian authors [8-10]. It is tempting to create new structural and functional materials using the biomimetic approaches. Biomimetics usually uses the principle of breaking up an object into component parts and their subsequent synthesis. That is, the material is first disassembled into its component parts which are identified and then reassembled on a matrix, or through a hypothetical self-assembly mechanism [11, 5]. It is believed that the more disassembly details there are and the cleaner the analyzed components are, the higher quality result one can achieve. However, this overlooks the destruction or elimination of some natural connections and structural features of the natural biocomposite. We should not forget the role of minor impurities, which can significantly affect the properties of a complex material. It is pertinent to mention here semiconductor technologies (impurity conductivity), alloys (alloying additives), glass production and ceramic materials, powder metallurgy.

Biologically induced mineralization can be achieved by means of an approach that is based on the selection and use of certain organic biomacromolecules, which play a crucial role in the nucleation and growth of bioorganic crystal-like substances. It was proposed to use such macromolecules as soft templates for inorganic components [1,5,6]. Wide application of such technologies in real practice requires unlimited resources of raw materials. These can be derived biotechnologically from renewable raw resources, or can be available in sufficient quantities as fossil resources in nature. For example, plants (trees and grasses such as spruce, larch, palm, bamboo, sedge) may contain up to $4 \%$ of silicon. Many cultivated plants, especially cereals (wheat, oats, barley, millet, rice, sugar beets, and others) can be classified as silicon-concentrating or silicophiles [12,13]. Minerals of organic origin such as oil, coal, shale, peat can also be used as raw materials.

Glass (Hyalospongia) or six-rayed sponges (Hexactinellida) are the most ancient, exclusively marine, multicellular animals that have survived to these days. Siliceous sponges are thought to have originated from the ancestors of multicellular animals (Urmetazoa) in the Precambrian [14]. These sponges with triaxonic, often hexactin (six), siliceous spicules of intracellular origin are known from the Proterozoic deposits of Australia, China and Mongolia; numerous spicules of hexactinellids are found in deposits, beginning in the Middle 
Cambrian, in many regions of the Earth [15]. The silico-organic spicules can be formatted in weak NAOH solutions [16].

The purpose of the present research is to develop a method for creating an organosilicon composite material based on soft incomplete alkaline etching of glass sponge spicules combined with directed application of external vectorizing forces (in this case, soft pulsating movements of a floating-up air bubble).

\section{Materials and methods}

We used the corporal spicules of the glass sponge Asconema setubalense (Hexasterophora, Lyssacinosida, Rossellidae). The material of sponges (kindly provided to us by K.R. Tabachnik) has been stored in the collection of the P.P. Shirshov Institute of Oceanolgy of the Russian Academy of Sciences (Moscow) for several decades. During storage the spicules have degraded and thus differed from the intact spicules from live freshly caught individuals.

Spicules were placed in a $1 \mathrm{M} \mathrm{NaOH}$ solution at $25{ }^{\circ} \mathrm{C}$ for a long time period (up to 6 months) under continuous rotational stirring. A bunch of glass sponge spicules (approximately $2-2.5 \mathrm{~cm}^{3}$ ) were placed in the center of a PS tube (height, $80 \mathrm{~mm}$; an inner diameter, $16 \mathrm{~mm}$ ) and a $1 \mathrm{M}$ aqueous solution of $\mathrm{NaOH}$ was then added to the spicules, so that a small air bubble (10 to $15 \mathrm{~mm}$ in diameter) remained under the stopper. The container with the object was rotated in a vertical plane at a speed that allowed the bubble to twice float up at most height to the very stopper in one rotation. Due to its oscillations in several planes during the free ascent, the air bubble serves as a kind of shape-forming gas device.

Raman microspectroscopy. Raman spectra were obtained using a Raman microscope spectrometer (in Via Reflex, Renishaw, UK) combined with an incident light microscope (Leica DM2500M, Leica-microsystems, Germany). Excitation was achieved with a diode laser at $532 \mathrm{~nm}$ and at a power of $1.0 \mathrm{~mW}$ at the object level and an exposure time of $10 \mathrm{sec}$ in 10-20 replicates. A laser spot of about $1.6 \mu \mathrm{m}$ in diameter on the slide was formed by an objective of microscope $(\times 50, \mathrm{NA}=0.6$, Leica $)$.

The elemental composition of spicules was studied using a Zeiss Evo 40 scanning microscope with an X-ray analyzer, using the INC software. The analyzed spicules were previously washed with a hydrogen peroxide solution. Elemental analysis was performed using the point method: several points forming a sector were chosen at a given part of a spicule, and the elemental composition of the spicule at these points was determined using the X-ray analysis.

For microscopic monitoring of the state of the material, part of it was periodically taken out of the container with alkali, washed in distilled water, and analyzed in light and scanning microscopes. For scanning microscopy, the material was sprayed with platinum and analysed with a Zeiss EVO 40 scanning electron microscope. For light microscopy, a Zeiss Axioplan2 imaging microscope (Zeiss, Germany) was used in a standard luminescent configuration in the UV, $\lambda$ vosb.max. $=365 \mathrm{~nm}$.

\section{Results}

The skeleton of the hexactinellid sponge Asconema setubalense is of the Lyssacine type with free spicules [17]. When dried, the sponge has the appearance of fiberglass felt (Fig. 1). The spicules of this glass sponge are of different thickness and morphology; each of them is a system of concentric thin layers that are laid around a central cylinder containing a square channel (Fig. 2A). After the spicules have been kept in an alkaline medium for several days, the material of the outer concentric layers is dissolved and passes into the silica solution. After 20-30 days, silica is in excess in the medium and it precipitates onto organic components, forming a network of thin organosilicon trabeculae that are 300-500 nm thick, by which individual spicules are fused into a single structure (Fig. 2B). Spicules at different stages of etching fastened with trabeculae of organic material. After 180 days in a $1 \mathrm{M}$ solution of $\mathrm{NaOH}$, granules consisting of silica and an organic matrix are observed in the trabeculae (Fig. 2B).

Subsequently, both processes - the dissolution and precipitation of silica - continue, resulting in structures of various shapes, for example, three-dimensional structures in the form of a cylinder with a very large inner surface area (Fig. 3A, 3B) or in the form of thin plates (Fig. 3C, 3D). Spicules are fastened by connective elements (trabeculae) that hold them together in a single flat "pseudo-fabric" non-monolithic structure. It takes the form of a three-dimensional body - a thin-walled tube tightly adjacent to the container walls, in which alkali treatment takes place. Glow in ultraviolet light indicates the presence of an organic component. In spaces between remains of etched spicules is a material based on silicates with organic cords embedded in it (Fig. 4A, 4B, 4C, 4D).

Tables 1 and 2 show the elemental analysis data of the initial spicules and the material allowing them to be fused together. There is no particular difference between them. Hence, the results confirm the conclusion on the identity of the materials of the spicules and the connective structures of the assembly. 


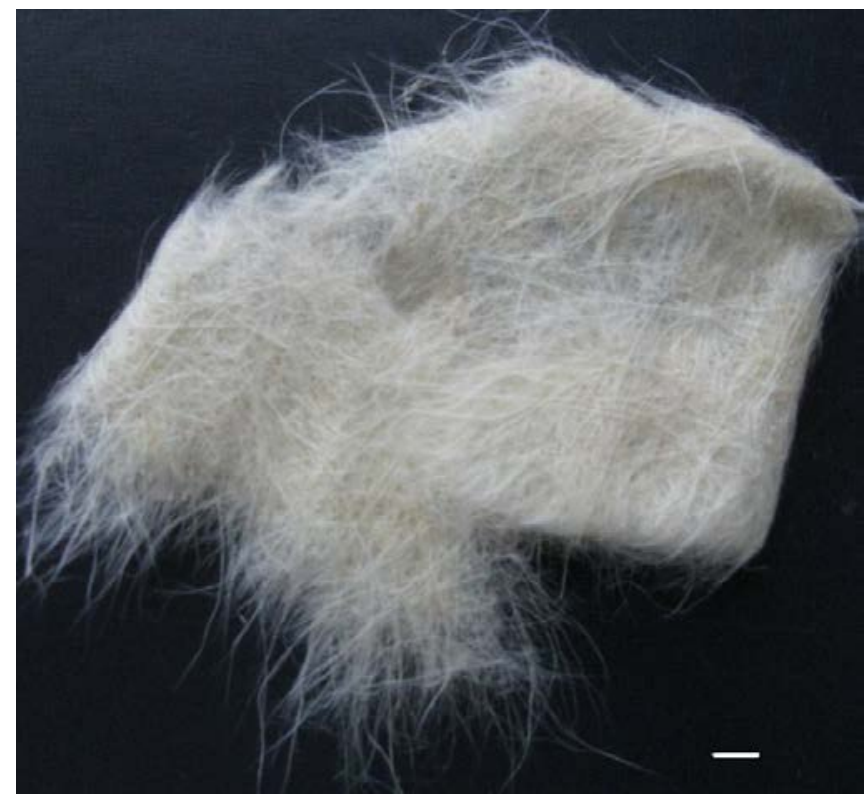

Fig. 1 Fragment of a dried glass sponge Asconema setubalense. Scale: 15 mm.
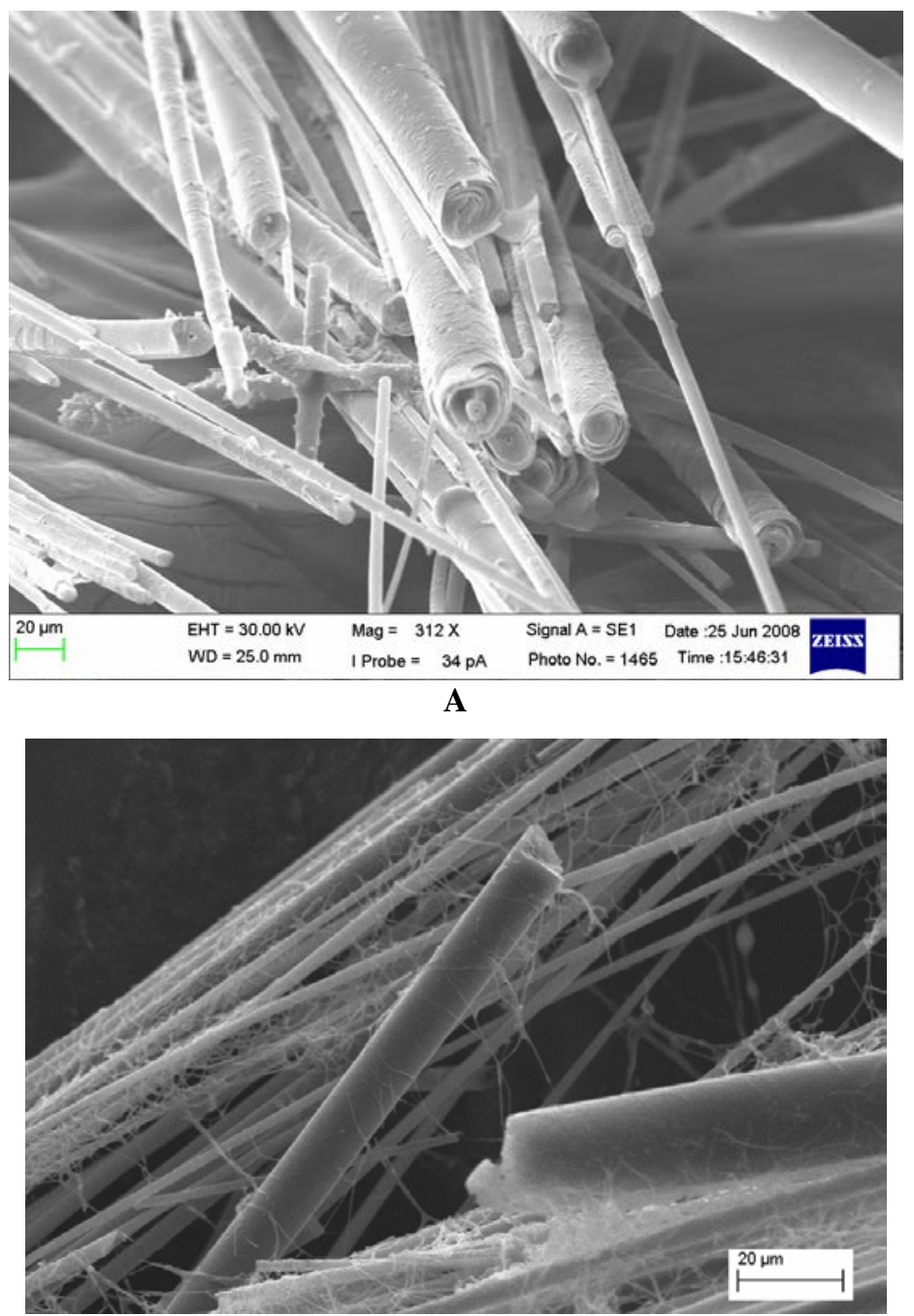

B

Fig. 2 SEM images of corporal spicules of A. setubalense. A - intact dry; B - after 60 days in $1 \mathrm{M}$ NaOH solution. Scale: $20 \mu \mathrm{m}$. 


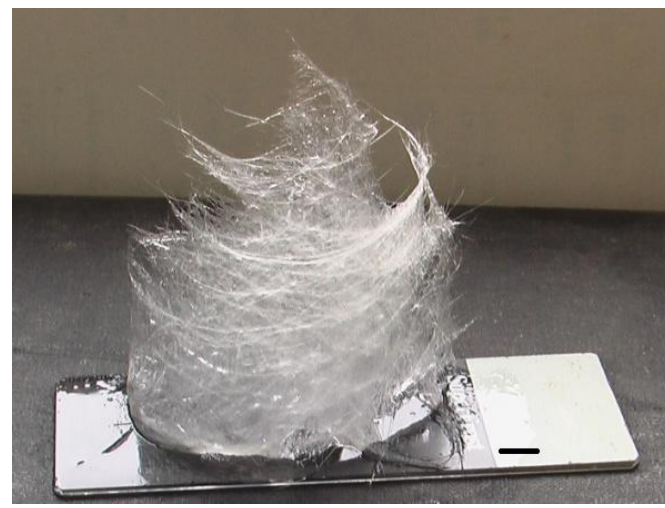

A

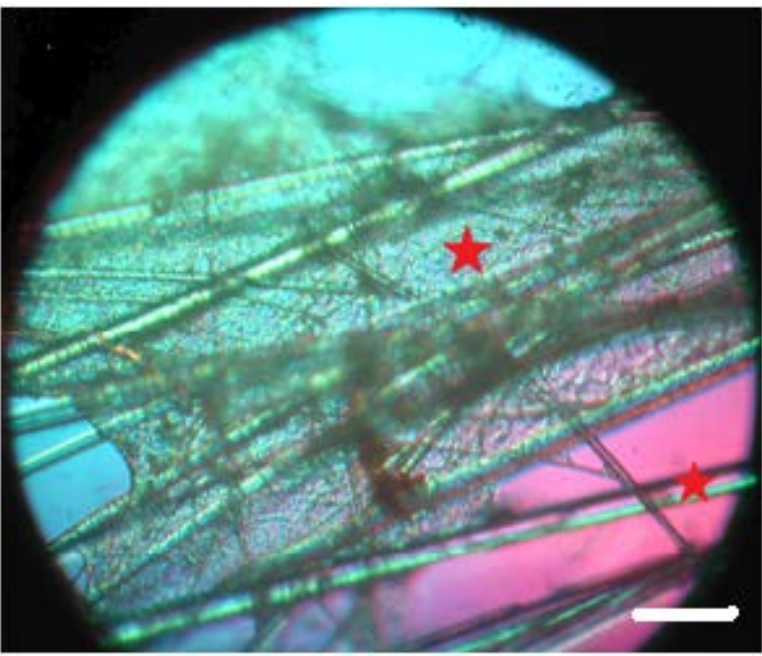

C

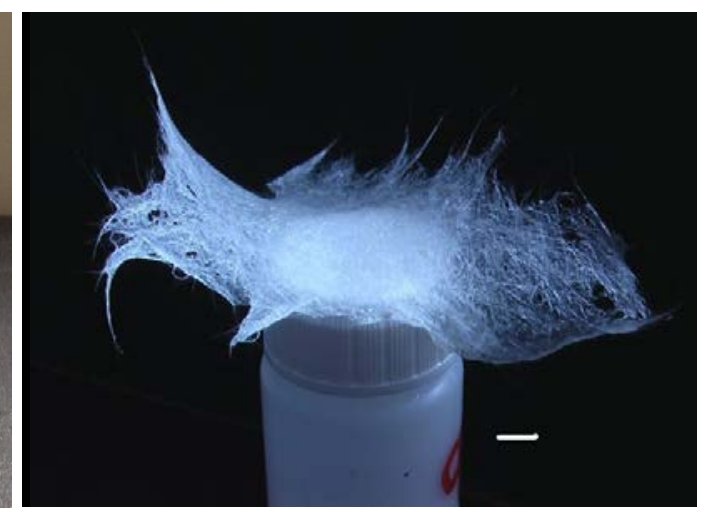

B

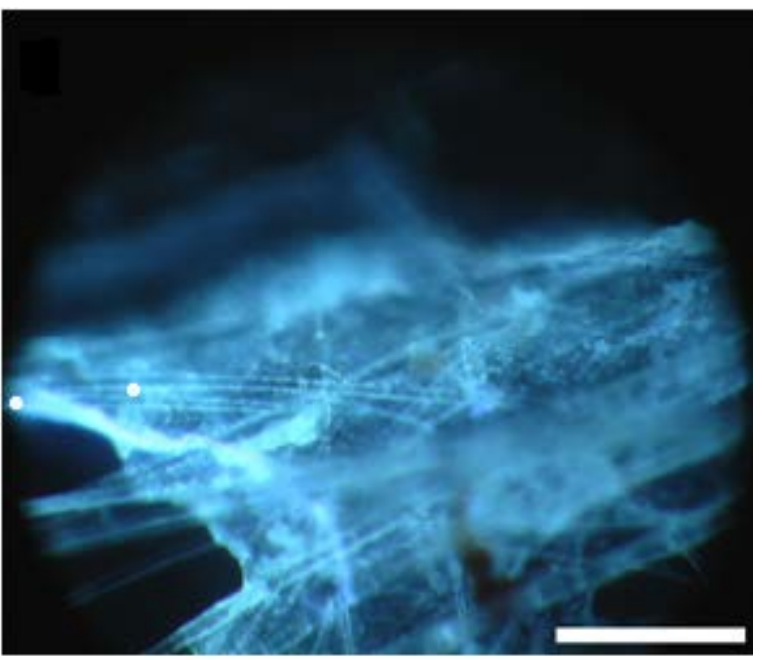

D

Fig. 3 Fused spicules of $A$. setubalense after 90 days in a 1 M NaOH solution. A - regular light; B - D ultraviolet light. $C$ - stars mark the registration sites of the Raman spectra. Scale: A, B - 10 mm, C - 50 $\mu \mathrm{m}, \mathrm{D}-100 \mu \mathrm{m}$.

Table 1. EDS analysis of initial crystal-like structures of the material.

\begin{tabular}{lllllllll}
\hline $\begin{array}{l}\text { Statistical } \\
\text { deviation }\end{array}$ & $\mathrm{O}$ & $\mathrm{Na}$ & $\mathrm{Al}$ & $\mathrm{Si}$ & $\mathrm{Ca}$ & $\mathrm{Zn}$ & $\mathrm{Ba}$ & Total \\
\hline Medium & 62.80 & 7.84 & 5.58 & 19.79 & 1.14 & 1.19 & 1.66 & 100 \\
Standart & 0.55 & 0.34 & 2.37 & 0.95 & 0.52 & 0.73 & 0.69 & \\
Maximum & 63.44 & 8.21 & 9.73 & 20.85 & 2.16 & 2.07 & 2.23 & \\
Minimum & 61.90 & 7.28 & 3.86 & 18.14 & 0.66 & 0.21 & 0.56 & \\
\hline
\end{tabular}

Table 2. EDS analysis of the residual material of spicules. A star symbol in Fig. 3 C.

\begin{tabular}{lllllllll}
\hline $\begin{array}{l}\text { Statistical } \\
\text { deviation }\end{array}$ & $\mathrm{O}$ & $\mathrm{Na}$ & $\mathrm{Al}$ & $\mathrm{Si}$ & $\mathrm{Ca}$ & $\mathrm{Zn}$ & $\mathrm{Ba}$ & Total \\
\hline Medium & 62.09 & 7.48 & 5.87 & 19.68 & 1.45 & 1.43 & 1.99 & 100 \\
Standart & 2.49 & 0.44 & 0.89 & 1.61 & 0.53 & 0.80 & 0.58 & \\
Maximum & 64.83 & 8.08 & 6.75 & 21.37 & 1.96 & 2.19 & 2.56 & \\
Minimum & 58.83 & 7.14 & 4.64 & 18.20 & 0.81 & 0.41 & 1.26 & \\
\hline
\end{tabular}

The Raman spectra of a fresh spicule sample showed a wide band centered at ca. $470 \mathrm{~cm}^{-1}$, which was attributed to the symmetric Si-O-Si stretching mode. The different angle of inclination of the curves is explained by the different levels of parasitic luminescence of the material, and therefore, by the different value of the constant component of the Raman signal (Fig. 5).

The spectra of the sponge samples showed a relatively sharp band at $967 \mathrm{~cm}^{-1}$, which may be assigned to silanol, as it is observed in hydrous amorphous silica materials. Amide I bands that can be assigned to the vibrational modes of the CONH group appeared in the spectrum of demineralized skeletal material at $1660 \mathrm{~cm}^{-1}$ (shoulders) and $1625 \mathrm{~cm}^{-1}$; and amide II bands, at ca. $1550 \mathrm{~cm}^{-1}$. Amide III bands can be found in the region 
between $1300 \mathrm{~cm}^{-1}$ and $1200 \mathrm{~cm}^{-1}$. Strong bands centered at $1062 \mathrm{~cm}^{-1}$ can be ascribed to the stretching of the C$\mathrm{O}-\mathrm{C}$ and $\mathrm{C}-\mathrm{O}$ modes of the protein. The intensity of the bands changed as a consequence of overlapping of the bands characteristic of chitin and silica between 1010 and $1040 \mathrm{~cm}^{-1}$. The rocking vibration of the methyl group can be found at $1385 \mathrm{~cm}^{-1}$.

The resulting composite material contains a mineral component of silica and an organic component. This is evidenced by the luminescence of the material caused by ultraviolet radiation (Fig. 3 B,C.D). Silica does not luminesce at this wavelength, but aromatic amino acids (phenylalanine, tryptophan, tyrosine) proteins, or chitinchitosan containing amino groups [6], do luminesce. The native spicules and the material subjected to alkaline etching were examined with a Raman microscope for the characteristics of their Raman spectra. The identity of the material of the spicules and the material that fastens them together at incomplete alkaline etching is confirmed by Raman spectra (Fig. 5).

Results of the elemental analysis of the initial spicules and the final crystal-like material are presented in Tables 1 and 2. The main elements in these structures are oxygen (O) and silicon (Si), nearly 62 and 20\%, respectively. In addition, the material contains metals $\mathrm{Na}, \mathrm{Al}, \mathrm{Ca}, \mathrm{Zn}$, and $\mathrm{Ba}$. There are no substantial differences between intact spicules and the resulting crystal-like material. The differences make up only a fraction of a percent. For example, the spicules contain $62.09 \%$ oxygen versus $62.80 \%$ in the resulting material, $7.48 \%$ sodium versus $7.84 \%$, etc.

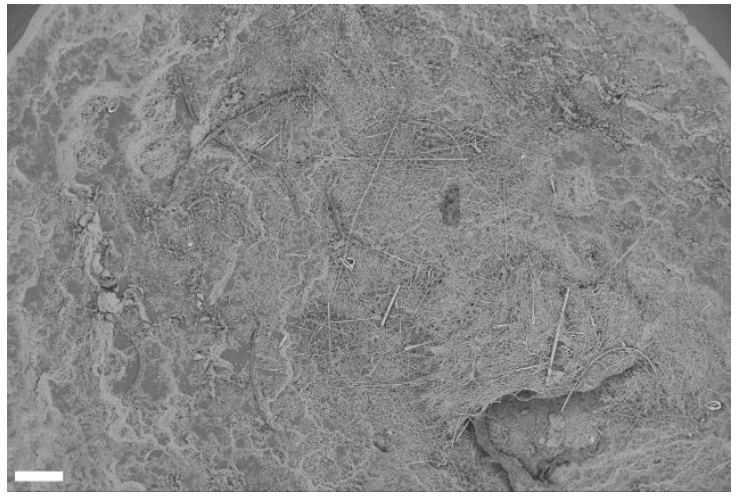

A

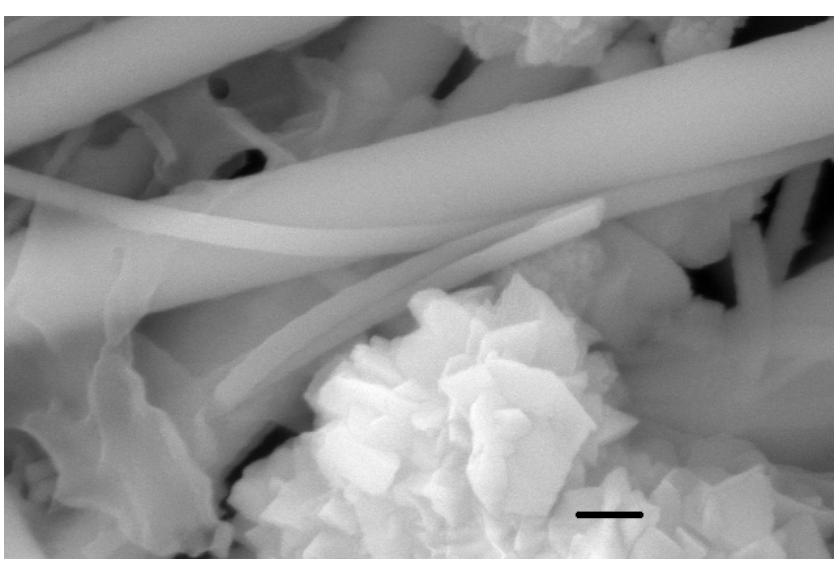

C

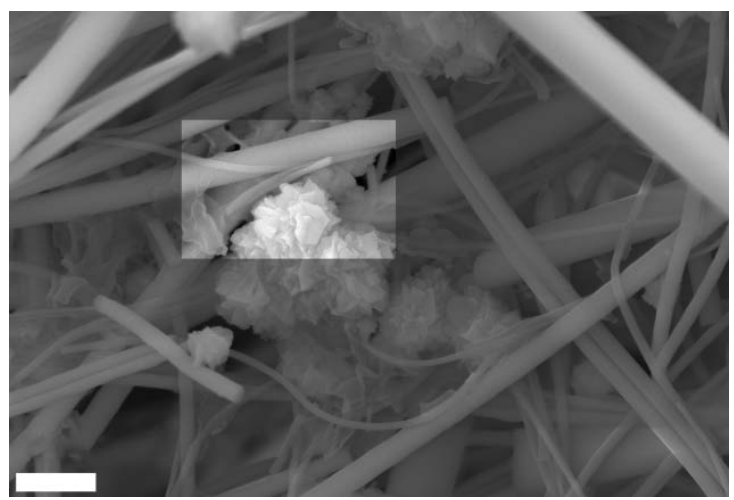

B

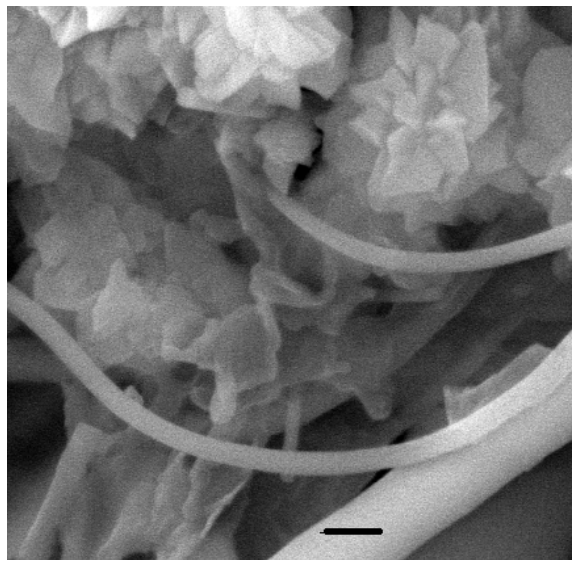

D

Fig. 4 SEM images of final composite material received from the "linear" spicules of A. setubalense. A general view; $B$ details of structure; C, D - enlarged fragment of $B$, see tape-like chitinous structures and organo-silicate crystal-like formations. Scale: A - $200 \mu \mathrm{m}, \mathrm{B}-1 \mu \mathrm{m}, \mathrm{C}, \mathrm{D}-\mathbf{0 , 5} \mu \mathrm{m}$.

\section{Discussion}

At present, the social demands for comfort, health, the quality of life and environment are increasing. This inevitably has raised a need for fundamentally new materials, including biomaterials, and products derived from them. Composite materials are increasingly used for creating various structures in various fields of industry, building, medicine, goods production etc. Layered materials with good mechanical properties and resistance to 
aggressive environments are the most promising as composite materials. In this regard, the methods of creating composite materials with a valuable set of properties have gained great importance [18].

Biomimetics (i.e. the study of the characteristics and natural mechanisms of the formation of biological tissues, composite materials, and structures) and the following creation of materials and structures with a given set of properties and, in particular, biocomposite nanomaterials, are yet to be developed despite the abundance of publications and the optimistic viewpoint of the authors of reviews. This is due to the lack of intelligible knowledge on the structural relationship between a biopolymer and mineral nanoparticles, which complicates the research and designing of materials with the required properties, as well as due to the lack of database required for a well-founded theory that would predict the properties of materials based on the properties of the biopolymer and mineral components of the designed biocomposite [19].
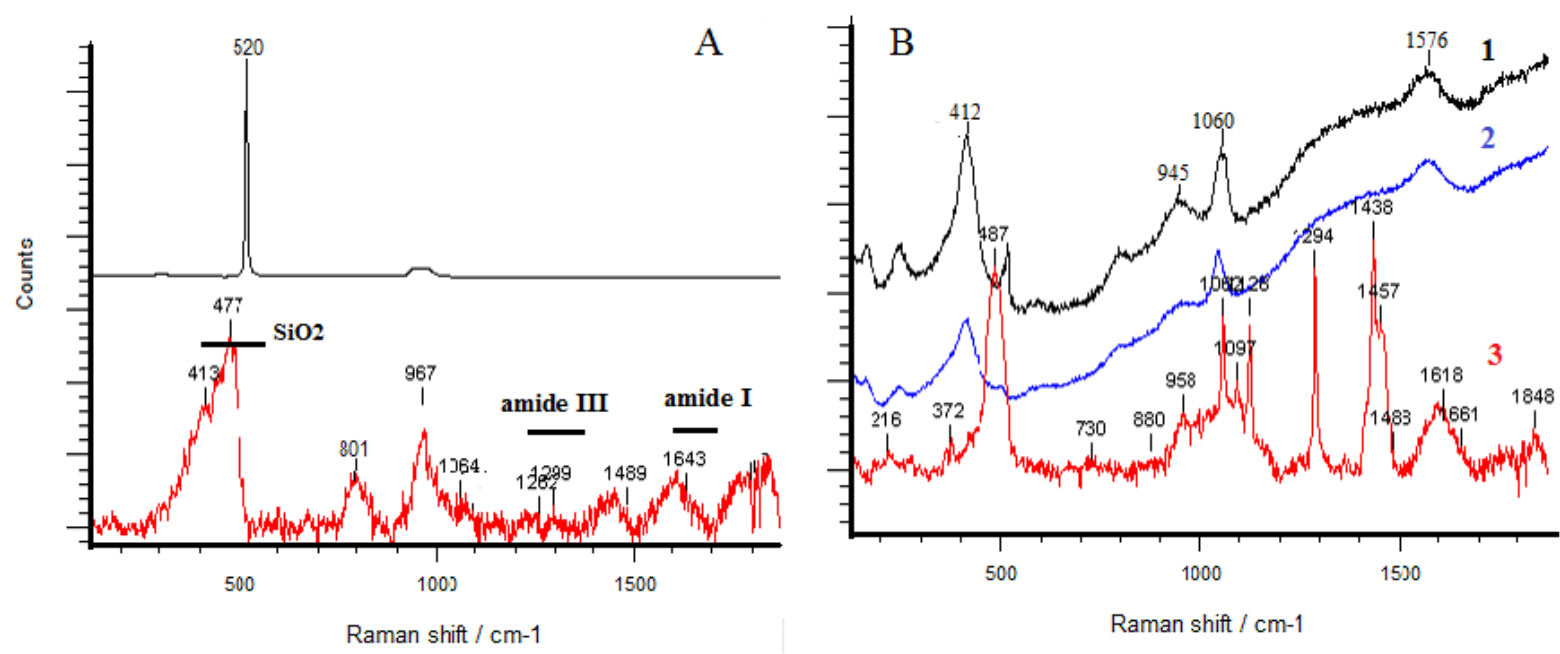

Fig. 5 Raman spectra of $A$. setubalense spicules (A) and finally received composite material (B). A - the upper curve represents the standard calibration spectrum for silicon, $520 \mathrm{~cm}^{-1}$. The bottom curve is a spectrum of an intact spicule. B - curve 1 corresponds to the site marked by the lower star in Fig. 3C; curve 2 corresponds to the site marked by the upper star in Fig. 3C; curve 3 - finally received composite material.

It is not clear to what extent the modern theory of composite materials is applicable to nanocomposites and especially to bionanocomposite materials [20]. It is appropriate to mention here the fate of bionics, a popular field of research in the 1960s-1970s that, based on applying principles found in living nature to the study and design of engineering systems, promised great prospects for various fields of science, technology, and national economy. Few people remember it now, and it is hardly possible to count the achievements.

The results we obtained in preliminary experiments show that the natural material of organosilicon spicules of sponges can be reformed through self-assembly into three-dimensional or flat structures. The data support the idea of assembly or, at least, embedding, as if onto a matrix, of composite blocks of monoliths. Silica is dissolved in an alkaline medium ( $\mathrm{NaOH}$ solution). In doing so, silica can be adsorbed on the released organic components, forming materials shaped like plates and three-dimensional structures. This is largely a hypothetical "mechanism" that requires further research and elaboration of methods and technologies. The structural features of the silica micro-network in the fused spicules frame and untreated spicules were essentially similar (Fig.4B), although being, at first glance, different from those of sponge's intact (untreated) spicules (Fig. 4A). We think that in the process of etching silicates pass into solution, and some organic material remains undissolved. With time, the ratio of organic material to silicon compounds increases, which is reflected in the Raman bands.

Comparison between the element composition of the native spicules to the material obtained through alkaline etching and mechanical action indicated that the composition of the composite material remains virtually unchanged as a result of the abovementioned procedures. The presence of metals interspersed in silica gives the latter the properties of a semiconductor, but not a dielectric [21]. Such microelement impurities affect the electrical properties of the spicules, as well as of the newly obtained material.

The presence of chitin in the final material is evident from graph 3 showing the Raman spectra with peaks between 1010 and $1040 \mathrm{~cm}^{-1}$ (Fig. 5). The Raman spectra of native spicules isolated from Asconema setubalense prior to the alkaline demineralization display intense bands of biosilica at 443, 480, 599, 640, and $805 \mathrm{~cm}^{-1}$. Bands of the organic matrices are visible in the range between 900 and $1800 \mathrm{~cm}^{-1}$. These bands are comparable 
with data described for standard chitin. Similar observations were previously reported for chitin from common sponges (Demospongiae) [5,6].

In our experiments, a floating air bubble plays a special role in the formation of composite material. The bubble moves by the forces of buoyancy and resistance, their interaction is expressed via surface tension. Taking into account the viscosity, the inertia of the surrounding fluid and buoyancy. While the air bubble is moving up, the height of the liquid column above it, and hence, the pressure of the liquid column, decrease with time. The pressure of the fluid flow in the front of the bubble and behind it differs. The flow ahead is laminar, the surface of the bubble is streamlined; behind it a low pressure area forms, the flow becomes turbulent, vortices with closed lines of flow appear. Therefore, the back wall of the bubble becomes quasi-flat; in some cases, small volumes of air come off it, which behave like elastic balls, because they are moving in flows of different pressure. Of particular interest are edge effects, in which the "crushing" of the smooth surface of the bubble takes place, i.e. the effect of the so-called standing capillary waves is observed. When meeting with irregular objects such as a group of thin flexible rods (a bundle of spicules), the air bubble pushes them toward the walls of the tube, "squeezing" between them. In doing so, part of the mineral components of the spicules becomes dissolved. The velocity of ascent decreases, the ascent mode becomes non-stationary. A combination of physical factors of such a regime (density, speed, pressure and time, concentration of substance, viscous stresses, volume forces, including buoyancy forces (gravity), surface tension coefficient) is, in our opinion, responsible for the formation of the shape. This allows a randomly oriented coil of spicules to be transformed into an ordered pseudo-woven 3D structure.

Thus, the linear spicules of siliceous sponges can be used to create both three-dimensional and 2D structures. The use of modified natural materials and - which is better - their biomimetic and the creation of new materials on this basis will help resolve some of the modern problems.

The glass sponge spicules can be used in the production of environmentally friendly materials, providing a wide range of substrates for creating biotech products [2, 6, 11], as well as biodegradable packaging materials, which are transformed into silicon sand on land and are dissolved without residue in the marine environment. In principle, it is possible to create structures with a "specified lifetime". As to the sources of materials for such technologies.

The situation with raw organic silicon material can be resolved easier due to large amounts of degraded fossil skeletons of siliceous sponges [14,15].

\section{Acknowledgements}

This research was partially supported by the Russian Science foundation: Project 19-14-00135.

\section{References}

[1] Xu AW, Ma Y, Cölfen H. Biomimetic mineralization. Journal of Materials Chemistry. 2007;17(5):415-449.

[2] Ehrlich H. Chitin and collagen as universal and alternative templates in biomineralization. International Geology Review. 2010;52(7-8):661-699.

[3] Sanchez C, Arribart H, Guille MM. Biomimetism and bioinspiration as tools for the design of innovative materials and systems. Nature materials. 2005;4(4):277-288.

[4] Nudelman F, Sommerdijk NA. Biomineralization as an inspiration for materials chemistry. Angewandte Chemie International Edition. 2012;51(27):6582-6596.

[5] Wysokowski M, Petrenko IA, Motylenko M, Langer E, Bazhenov VV, Galli R, Stelling AL, Kljajić Z, Szatkowski T, Kutsova VZ, Stawski D, Jesionowski T. Renewable chitin from marine sponge as a thermostable biological template for hydrothermal synthesis of hematite nanospheres using principles of extreme biomimetics. Bioinspired Materials. 2015; 1(1): 12-22.

[6] Ehrlich H, Shaala LA, Youssef DT, Żółtowska-Aksamitowska S, Tsurkan M, Galli R, Meissner H, Wysokowski M, Petrenko I, Tabachnick KR, Ivanenko VN. Discovery of chitin in skeletons of nonverongiid Red Sea demosponges. PloS ONE. 2018;13(5):e0195803.

[7] Müller WEG, Grachev MA. Biosilica in Evolution, Morphogenesis, and Nanobiotechnology: Case Study Lake Baikal. Springer Science \& Business Media. 2009. p.421.

[8] Cattaneo-Vietti R, Bavestrello G, Cerrano C, Sarà M, Giovine M, E. Gaino E. Optical fibers in an Antarctic sponge. Nature. 1996; 383: 397-398. doi: 10.1038 / 383397b0

[9] Mayer G. Rigid biological systems for composites. Science. 2005; 310 (5751): 1144-1147.

[10] Kulchin YuN, Bagaev SN, Bukin OA, Voznesensky SS, Drozdov AL, Zinin YuA, Nagorny IG, Pestryakov EV, V.I. Trunov VI. Photonic Crystals Based on Natural Biominerals of Oceanic Origin. Pis'ma ZhTF. 2008; 34(15): 1-7. 
[11] Ehrlich H, Worch H. Sponges as natural composites: from biomimetic potential to development of new biomaterials. Porifera Research: Biodiversity, Innovation and Sustainability. Museu Nacional, Rio de Janeiro, Brasil. 2007: 303-312.

[12] Voronkov MG, Kuznetsov IG. Silicon in Nature - Novosibirsk. Science. 1984.p.155. (In Russian)

[13] Voronkov MG, Zelchan GI, Lukevitz EE. Silicon and life. Biochemistry, toxicology and pharmacology of compounds of silicon. Edited K. Ruhlmann. Akademie Verlag, Berlin 1975; Peris 48: 370. (In German)

[14] Müller WE, Li J, Schröder HC, Qiao L, Wang X. The unique skeleton of siliceous sponges (Porifera; Hexactinellida and Demospongiae) that evolved first from the Urmetazoa during the Proterozoic: a review. Biogeosciences. 2007; 4: 219-232.

[15] Krautter M. Fossil Hexactinellida: an overview. In: Systema Porifera. Springer, Boston, MA.p. 1211-1223

[16] Ehrlich H, Ereskovskii AV, Drozdov AL, Krylova DD, Hanke T, Meissner H, Heinemann S, Worch H. A modern approach to demineralization of spicules in glass sponges (Porifera: Hexactinellida) for the purpose of extraction and examination of the protein matrix. Russian Journal of Marine Biology. 2006;32(3):186193.

[17] Drozdov AL, Tabachnik KR, Ekert K, Tyurin SA. Skeleton organization and typology of axial channels of axial filaments of glass sponge spicules. In: Perspective directions of development of nanotechnology in the Far East Branch of the Russian Academy of Sciences. Ed. Kulchin YuN. Vladivostok 2009; 2: 87-99. (In Russian)

[18] Richardson SG. Industrial composite materials. Moscow: Chemistry. 2002.p.320. (In Russian)

[19] Balazs AC, Emrick T, Russell TP. Nanoparticle polymer composites: where two small worlds meet. Science. 2006; 314(5802): 1107-1110.

[20] Winey KI, Vaia RA. Polymer nanocomposites. MRS Bull. 2007; 32: 314-319. doi: 10.1557/mrs2007.229.

[21] Drozdov AL, Andreykin NA, Dorofeev AG Structure and physico-chemical properties of organosilicon crystal-like composite spicules of the glass sponge Hyalonema sieboldi. Materials Research Bulletin. 2018; 105: 372-376.

(C) 2019 by the author(s). This work is licensed under a Creative Commons Attribution 4.0 International License (http://creativecommons.org/licenses/by/4.0/). Authors retain copyright of their work, with first publication rights granted to Tech Reviews Ltd. 